\title{
The Positive Aspect of Unresolved Questions: The Example of Perilymphatic Fistula
}

This is the second issue of 'Controversies in ORL'. It is devoted to the physiopathology, diagnosis and treatment of perilymphatic fistula (PLF). The issue does not pretend to offer definitive answers to the many questions about the existence of PLF or to establish guidelines for its diagnosis and treatment. Such an attitude would be presumptuous in view of the many uncertainties regarding the physiopathology, the diagnostic criteria, the surgical findings and the benefit of the proposed therapies. This issue does not intend either to bring about a consensus, a concept which is polite in politics but meaningless in science. The purpose of this issue is to raise and illuminate the many unresolved aspects of PLF diagnosis and treatment. Facing these limited ambitions, the ENT practitioner may feel disappointed and consider that it is not even worth reading the journal. On the contrary, he should rather react positively. Such a controversial topic confirms that medicine remains an art and that complex diagnostic processes cannot be oversimplified and reduced to standardized guidelines.

Controversial issues are interesting in that they reinforce the role of the physician and enhance his responsibility. He has to develop his own concept by choosing among many existing more or less reasonable theories. When renowned experts cannot agree on a topic such as PLF, each practitioner shares then the responsibility to keep improving our understanding of the problem. Each otologist must therefore adhere to strict criteria of assessment and be ready to modify his opinion according to new data. An extensive knowledge of the many hypotheses of the physiopathology of PLF, as well as a full understanding of the controversies regarding the diagnostic proce- dures and treatment results should help the clinician to critically review his own concept of the disease and to better analyse his results. In this regard, we hope this issue of the journal will be useful.

Prof. Nelson Kiang ${ }^{1}$ greatly contributed to the elaboration of the format of these special issues designed to encourage discussion and critical thinking on currently controversial issues in clinical otorhinolaryngology. We are fully aware that he largely contributed to the success of the first debate dedicated to 'Otitis media in Children' which appeared earlier in March. We wish to thank him for his enthusiastic support and his many interesting suggestions.

Jean-Philippe Guyot, Geneva

Professor of Physiology emeritus, Department of Otology and Laryngology, Harvard Medical School; Eaton-Peabody Professor emeritus, Harvard-MIT Division of Health Sciences and Technology and Department of Brain and Cognitive Sciences, Massachusetts Institute of Technology; former Director, Eaton-Peabody Laboratory, Massachusetts Eye and Ear Infirmary; Neurophysiologist emeritus, Neurology Service, Massachusetts General Hospital; former Director, Speech and Hearing Sciences Graduate Program, Harvard-MIT Division of Health Sciences and Technology, Massachusetts Institute of Technology. 